\title{
Ubi solitudinem faciunt, pacem appellant! [1]
}

Le $1^{\text {er }}$ janvier 2007 verra l'introduction simultanée du nouveau code pénal des mineurs et du nouveau droit des sanctions du Code pénal des adultes. Le code des mineurs prévoit la possibilité de sanctions plus lourdes (jusqu'à 4 ans de privation de liberté), plus de soins, plus de mesures éducatives dans des lieux adaptés (à créer) mais aussi plus d'expertises pour lesquelles il faudra former des experts compétents.

Quant au CPS adulte, la partie générale révisée introduit plusieurs changements dont les effets seront majeurs sur le corps médical [2]. Il prévoit moins d'incarcérations en dessous de 6 mois et donc un plus grand choix d'alternatives à ce type de peine privative de liberté. En fonction de l'usage qui en sera fait par les magistrats, les personnes souffrantes risquent d'être détenues soit plus soit moins longtemps qu'aujourd'hui. Il prévoit aussi des expertises obligatoires pour ordonner des mesures. L'expert doit être nouveau pour les internements ordinaires mais il en faut deux, nouveaux également, pour les internements à vie. $Y$ aura-t-il suffisamment d'experts psychiatres «neufs, spécialisés et expérimentés» pour faire face à ces nouvelles exigences? Ce n'est pas certain. Le nouveau code prévoit aussi une commission fédérale chargée d'évaluer les possibilités thérapeutiques chez les personnes internées à vie. Nous ne savons pas s'il y aura des «progrès scientifiques suffisants» pour avoir l'autorisation de soigner ces condamnés, auxquels on ne proposera rien dans le cas contraire! Quant aux lieux d'exécution des mesures, ils devront être «adaptés» et «spécialisés». Pour les mesures thérapeutiques institutionnelles il faudra prévoir des «établissements psychiatriques appropriés». Actuellement, ce type de lieu est très rare. Quant à l'internement, il devrait s'effectuer dans des établissements «fermés, sécurisés, avec une prise en charge psychiatrique». Aucun établissement de ce type n'est encore construit ni le personnel formé. Pour l'internement (art. 64 nCPS), il est prévu que, pour les délinquants souffrants «d'un grave trouble mental chronique ou récurrent», la peine privative de liberté précède l'internement. Il en va de même pour l'internement à vie des délinquants «durablement non amendables». Dans quelles structures, avec quels professionnels et avec quels moyens sera soignée cette catégorie de détenus? Cela reste obscur! Rappelons que l'exécution des peines pour les détenus souffrant de graves troubles psychiques doit se faire en conformité avec les Règles pénitentiaires européennes; celles-ci stipulent que les «personnes souffrant de maladies mentales et dont l'état de santé est incompatible avec la détention en prison devraient être détenues dans un établissement spécialement conçu à cet effet».
La justice s'est donc dotée d'un instrument de répression, d'ordre et de pacification précis qui interpelle aussi les soignants. Afin que la prison ne devienne pas le dernier lieu de gestion et de contrôle des exclusions, la FMH tient à rappeler les préliminaires à toutes interventions du corps médical pour une population carcérale de plus en plus jeune, au statut économique et social précaire et souffrant de troubles tant somatiques que psychiques (jusqu'à $30 \%)$.

Premièrement quelle que soit la catégorie de la population concernée ainsi que le lieu de pratique, la FMH demande et soutient un accès aux soins de qualité pour tous. En témoigne la décision du Comité central de financer une recherche de la Conférence suisse des médecins pénitentiaires sur le respect des directives de l'Académie Suisse des Sciences Médicales concernant l'exercice de la médecine auprès des personnes détenues. La FMH soutient également la formation en médecine et psychiatrie forensique, que ce soit dans le domaine des soins ou dans celui des expertises.

Soigner n'est compatible ni avec le «furor sanandi» qui conduit à appliquer de force certains types de soins, ni avec le concept de contrôle social. La généralisation des exigences de transparence actuellement prônée conduit pourtant à une ingérence de plus en plus forte du judiciaire dans les soins. C'est pourquoi, pour garantir l'intimité et le secret de la consultation, le lieu de soin doit être défendu comme distinct et indépendant du lieu de surveillance. Indépendant mais articulé; c'est pourquoi la FMH soutiendra et défendra une pratique thérapeutique autonome qui s'exerce tant dans l'intérêt du sujet que dans celui de la collectivité.

Comme médecins, il nous appartient de défendre un acte thérapeutique responsable fondé sur une éthique de la conséquence. Cela implique de ne pas se situer uniquement dans une logique causale, dans l'immédiateté de la réponse à une demande, que celle-ci vienne du patient et/ou de la collectivité. Dans son discours aux médecins de 1966, Jacques Lacan rappelle que «C'est dans le registre du mode de réponse à la demande du malade qu'est la chance de survie de la position proprement médicale» et non pas "comme agent de contrôle social ou encore agent de distribution de la santé». "La signification de la demande est la dimension où s'exerce à proprement parler la fonction médicale [...] à savoir dans la faille qui existe entre la demande et le désir». Dans le champ de la médecine et de la psychiatrie forensique, une clinique orientée par une éthique de la conséquence est multidisciplinaire mais surtout elle 
consiste à ne pas démissionner face à la subjectivité de la folie et de faire «en-corps» avec l'insensé.

Avec l'appui du citoyen, la justice et le domaine d'application des peines se sont dotés d'instruments de répression certes nécessaires mais aussi très idéaux quant à leur application. Il s'agit de faire en sorte que, de cette volonté de pacification - de l'inquiétude comme de l'insécurité sociale -, ne surgisse pas un désert pour les soignants et leurs patients.

Dr R. Raggenbass Psychiatre psychothérapeute Membre du Comité Central de la FMH Responsable du Domaine Santé Prévention Membre du Comité de la Société Suisse de Psychiatrie Forensique

\section{Références}

1 «à où ils ont laissé un désert, ils disent qu'ils ont apporté la paix!» Tacite, Vie d'Agricola, XXX.

2 Je remercie le Professeur Nicolas Queloz, professeur au Département de droit pénal et de criminologie de l'Université de Fribourg, pour la mise à disposition de son exposé.

Celui-ci ainsi que les autres travaux des cinquièmes journées de formation du personnel pénitentiaire qui ont eu lieu du 7 au 9 novembre 2006 seront publiés sous: de Sinner P, Queloz N, et al (éds.). Prison-asile? La problématique des détenus souffrant de troubles psychiques. Berne: Stämpfli; 2007 (à paraître) 